
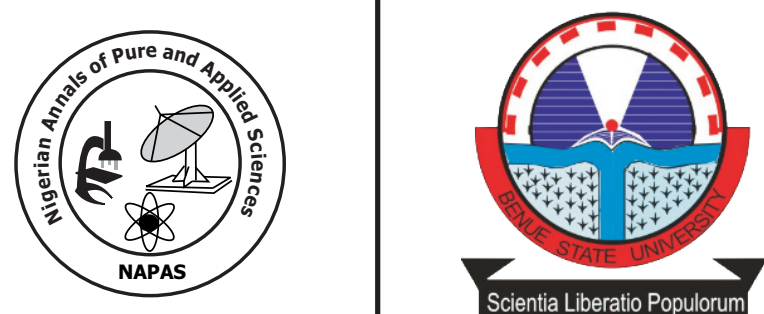

\title{
Deriving Cauchy's Integral Formula Using
} Division Method

Egahi, M., Ogwuche, I.0.; and Ode, J.

Department of Mathematics and Computer Science

Benue state University, Makurdi

Corresponding author:egahimusa@yahoo.com, musaegahi@gmail.com

\section{Abstract}

Cauchy's integral theorem and formula which holds for analytic functions is proved in most standard complex analysis texts. The nth derivative form is also proved. Here we derive the nth derivative form of Cauchy's integral formula using division method and showed its link with Taylor's theorem and demonstrate the result with some polynomials.

Key words: Cauchy's Integral Formula, Cauchy's Theorem, Analytic Function, Division, Polynomials. 


\section{Introduction}

Cauchy's theorem is regarded as a basic result in complex analysis as analysed by Conway (1986). The theorem along with the Cauchy's integral formula is powerful tool for contour integration and some types of real integrals when combined with the method of residues. Through a very simple application of Cauchy's theorem, it becomes possible to represent analytic functions as line integrals which the variable $\mathrm{z}$ enters as a parameter (Ahlfors, 1979). It is this representation that is known as Cauchy's integral formula and has numerous important applications. McCharty (1975) usedCauchy's integral formulato prove Cayley-Hamilton theorem and more importantly, it enables us to study the local properties of an analytic function in great details.

The original result of Cauchy was published in 1825 in which he used the idea of calculus of variation, and since then the theorem has witnessed improvement by various mathematicians. Cauchy himself gave a second prove of the theorem in 1846 using the concept of exact differentials together with Green's theorem. After Cauchy, the next version of the proof was published by Riemann in 1953. Riemann broadened the scope of the theorem to include all continuous functions with continuous derivative on and inside a simple closed curve. Many other significant developments were made during the remainder of the nineteenth century (Alpay, 2015)

Goursatin 1900 drew attention of mathematicians back to the basics of the theory when he proved Cauchy's theorem without the assumption of a continuous derivative. This proof introduced a new method of approach, which is working directly with the integral rather than separating it into its two real components (Scott, 1978).

Shortly, after Goursat's proof was published, Moore in the same year, endeavoured to rigorize the argument. Though Moore somewhat restricted the class of functions for which the theorem applied by imposing a special condition on them, he was careful to include the concepts of rectifiability for the curve and simple connectivity for the domain. Motivated by Moore, Pringsheim published a proof of the theorem within the next year. It was a simple proof for simple rectifiable curves, the idea being to prove the theorem for triangles and then apply the result with some limit considerations to approximate the integral along a closed curve by one along an inscribed polygon (Scott, 1978)

The final stage of the proof was in the mid twentieth century. This time the contributions to the proof were topological in nature. The last analytical development in the proof of the theorem was by Dixon (1971). He used a local version of the integral theorem, properties of analytic functions were developed and using these properties, the Cauchy's integral formula and ultimately, the Cauchy's integral theorem were proved. The significance of Dixon's proof was that it broadened the scope of the theorem even further by including all domains in its hypothesis (Scott, 1978).

More recent authors like Dyer and Edmunds (2014), John and Russell (2011) and Juan (2016) based their proof on the homology version of Dixon. We recall the theorem here.

\section{Cauchy's Integral Theorem:}

If $f(z)$ is analytic inside and on a contour $C$, then the integral of $f(\mathrm{z})$ around the curve is zero, where $\mathrm{C}$ is oriented in the positive sense (counterclockwise). That is

$$
\int_{C} f(z) d z=0
$$

For the application, it is very important that the conclusion of Cauchy's theorem remains valid under the weaker condition which we state as a theorem below:

\section{Theorem 1:}

Let $f(\mathrm{z})$ be analytic in a region $\Delta$ obtained by omitting a finite number of points $z_{i}$ from an open disc $\Delta$. If $f(z)$ satisfies the condition $\lim _{z \rightarrow z_{i}}\left(z-z_{i}\right) f(z)=0$ for all $i$ then (1) holds for any closed curve $\mathrm{C}$ in $\Delta^{\prime}$. (Ahlfors, 1979).

To get the Cauchy's integral formula, let $f(z)$ be analytic in an open disc $\Delta$ and consider a closed curve $C$ in $\Delta$ and a point $a$ in $\Delta \Delta$ which does not lie in C. Then apply Cauchy's theorem to the function $F(\mathrm{z})$ $=(f(z)-f(\alpha)) /(z-\alpha)$ which is analytic for $z \neq \alpha$ but satisfy Theorem 1 . For

$$
\lim _{z \rightarrow a} F(z)(z-a)=\lim _{z \rightarrow a}(f(z)-f(a))=0
$$

which lead to the conclusion that

$$
\int_{C} \frac{f(z)-f(a)}{z-a} d z=0
$$

From (2) above, we have

$\int_{C} \frac{f(z)}{z-a} d z=f(a) \int_{C} \frac{d z}{z-a}$

The integral $\int_{C} \frac{d z}{z-a}$ is by definition $n(\mathrm{C}, a) 2 \pi i$

where $n(\mathrm{C}, a)$ is the index number of the point $a$ with respect to the curve $\mathrm{C}$ or the winding number of $\mathrm{C}$ with respect to $a$. The most common application is the case where $n(\mathrm{C}, a)=1$, so that 


$$
f(a)=\frac{1}{2 \pi i} \int_{C} \frac{f(z)}{z-a} d z
$$

is the Cauchy's integral formula. Now $a$ can assume any value, say $\mathrm{z}$ in $\mathrm{C}$ so that

$$
f(z)=\frac{1}{2 \pi i} \int_{C} \frac{f(s)}{s-z} d s
$$

With (3)' we can now prove that analytic functions have derivatives of all orders (Brown and Churchill, 2009). The proof uses the fact that (3) is analytic and differentiated to give

$$
f^{\prime}(z)=\frac{1}{2 \pi i} \int_{C} \frac{f(s)}{(s-z)^{2}} d s
$$

byusing the difference quotient

$$
\frac{f(z+\Delta z)-f(z)}{\Delta z}=\frac{1}{2 \pi i} \int_{c}\left(\frac{1}{s-z-\Delta z}-\frac{1}{s-z}\right) \frac{f(s)}{\Delta z} d s
$$

which gives (4) as $\Delta z$ approaches zero. Further $f$, $(z)$ is analytic so we differentiate to get

$$
f^{\prime \prime}(z)=\frac{2}{2 \pi i} \int_{C} \frac{f(s)}{(s-z)^{3}} d s
$$

Again, the difference quotient is

$$
\begin{gathered}
\frac{f^{\prime}(z+\Delta z)-f^{\prime}(z)}{\Delta z}=\frac{1}{2 \pi i} \int_{C}\left(\frac{1}{(s-z-\Delta z)^{2}}-\frac{1}{(s-z)^{2}}\right) \frac{f(s)}{\Delta z} d s \\
=\frac{1}{2 \pi i} \int_{C}\left(\frac{2(s-z)-\Delta z}{(s-z-\Delta z)^{2}(s-z)^{2}}\right) \frac{f(s)}{\Delta z} d s
\end{gathered}
$$

which establishes (5) as $\Delta Z$ approaches zero.

This process continues and the nth derivative is given by

$$
f^{n}(z)=\frac{n !}{2 \pi i} \int_{C} \frac{f(s)}{(s-z)^{n+1}} d s \quad n=1,2, \ldots
$$

is the nth derivative form of the Cauchy's integral formula.If we allow that $f^{0}(z)$ denotes $f(z)$, and since $0 !=1$, we can write (3) ${ }^{\prime}$ and (6) together as

$$
f^{n}(z)=\frac{n !}{2 \pi i} \int_{C} \frac{f(s)}{(s-z)^{n+1}} d s \quad n=0,1,2, \ldots
$$

It is this integral formula we want to establish using division method and demonstrate the result with polynomials (without loss of generality), which are entire functions in the complex plane. Another reason for the choice of polynomials is that they have power series of finite degree $n$. Note that we can rewrite (7) as

$$
\frac{f^{n}(z)}{n !}=\frac{1}{2 \pi i} \int_{C} \frac{f(s)}{(s-z)^{n+1}} d s \quad n=0,1,2, \ldots
$$

Our observation is that the left hand side of (8) is exactly the same as the value of the remainder if we divide $f(z)$ by $z-a$ for any fixed $a$ and variable $z$. Also, for an application of (8), we note that if $C:|z-a|=r>0$ and $f(\mathrm{z})=1$,

$$
\int_{C} \frac{d z}{z-a}=2 \pi i \text { and } \int_{C} \frac{d z}{(z-a)^{n+1}}=0, \quad n=1,2,3, \ldots
$$

\section{Methodology}

The method we use is to divide the given analytic function, which in this case is a polynomial of degree $n$ by $(\mathrm{z}-a)$, where $a$ belong to the region of analyticity of belong to the region of analyticity of $f$. We can then write $f$ as a product of $(\mathrm{z}-a)$ and the quotient plus the remainder. The next step is to divide the quotient by $(\mathrm{z}-a)$. Again the quotient can be written in terms of $(\mathrm{z}-a)$ and a second quotient plus a second remainder. $f$ is then written out to include the two quotients and the two remainders. We continue the process until the nth step and finally write out $f$ in terms of the divisors, quotients and the remainders. The next thing is to divide through by $(\mathrm{z}-a)^{n+1}$ and then integrate around the curve $\mathrm{C}$ where $C:|z-a|=$ $r>0$. Our claim is that the remainders are precisely the Taylor series coefficients which are easily calculated.

We proceed formally by choosing an arbitrary polynomial of degree $n$ and divide it by $(\mathrm{z}-$ a) Let the polynomial be $P_{n}(\mathrm{Z})$ so that 


$$
P_{n}(z)=a_{0}+a_{1} z+a_{2} z^{2}+\cdots+a_{n} z^{n}
$$

As we divide $P_{n}(z)$ and its quotients repeatedly by $z-a$ and taking note of the remainders, we have the following:

At the $\mathrm{n}^{\text {th }}$ division, we have

$$
\begin{array}{r}
a_{0}+a_{1} z+a_{2} z^{2}+\cdots+a_{n} z^{n}=(z-a) P_{n-1}(z)+R_{1} \\
=(z-a)^{2} P_{n-2}(z)+R_{2}(z-a)+R_{1} \\
=(z-a)^{3} P_{n-3}(z)+R_{3}(z-a)^{2}+R_{2}(z-a)+R_{1}
\end{array}
$$

$$
\begin{aligned}
a_{0}+a_{1} z+a_{2} z^{2}+\cdots+a_{n} z^{n}= & a_{n}(z-a)^{n}+R_{n}(z-a)^{n-1}+R_{n-1}(z-a)^{n-2}+\cdots \\
& +R_{3}(z-a)^{2}+R_{2}(z-a)+R_{1}
\end{aligned}
$$

Letting $P_{n}(z)=f(z)$ and dividing through by $(z-a)^{n+1}$ we will get

$$
\frac{f(z)}{(z-a)^{+1}}=\frac{a_{n}}{(z-a)}+\frac{R_{n}}{(z-a)^{2}}+\frac{R_{n-1}}{(z-a)^{3}}+\cdots+\frac{R_{3}}{(z-a)^{n-1}}+\frac{R_{2}}{(z-a)^{n}}+\frac{R_{1}}{(z-a)^{n+1}}
$$

Integrating along $C:|z-a|=r, r>0$, the Cauchy's integral formula emerges. In fact, we can say more that

$$
\frac{1}{2 \pi i} \int_{C} \frac{f(z)}{(z-a)^{n+1}}=a_{n}=\frac{f^{n}(a)}{n !}
$$

which is already an existing result (Needham, 1997). All these results become simpler and clearer from just simple division.

\section{Results}

In this section, we demonstrate the methods described above on some polynomials.

\section{Example}

Consider the polynomial $f(z)=3 z^{2}-\frac{5}{2} z+\frac{1}{2}$ and we want to take $a=\frac{i}{2}$. We do the following: $3 z+(3 i-5) / 2$

$$
\begin{gathered}
z-\frac{i}{2} 3 z^{2}-\frac{5}{2} z+\frac{1}{2} \\
-\left(3 z^{2}-\frac{3 i}{2} z\right) \\
\left(\frac{3 i-5}{2}\right) z+\frac{1}{2} \\
-\left[\left(\frac{3 i-5}{2}\right) z-\frac{i}{2}\left(\frac{3 i-5}{2}\right)\right] \\
\frac{-1-5 i}{4}
\end{gathered}
$$

The remainder is $(-1-5 i) / 4$. This remainder is $f(a)=f(i / 2)$. Since

We can then write

$$
f\left(\frac{i}{2}\right)=3\left(\frac{i}{2}\right)^{2}-\frac{5}{2}\left(\frac{i}{2}\right)+\frac{1}{2}=-\frac{1}{4}-\frac{5 i}{4}
$$

$$
3 z^{2}-\frac{5}{2} z+\frac{1}{2}=\left(z-\frac{i}{2}\right)\left(3 z+\frac{3 i-5}{2}\right)+\frac{-1-5 i}{4}
$$

Now we divide through by $z-i / 2$ so that

$$
\frac{f(z)}{z-i / 2}=3 z+\frac{3 i-5}{2}+\frac{(-1-5 i) / 4}{z-i / 2}
$$

We now integrate both sides around a circle that contain $i / 2$ in its interior.

Let $C:|z-i / 2|=r, 0<r<\infty$. Then

$$
\int_{C} \frac{f(z)}{z-\frac{i}{2}} d z=\int_{C}\left(3 z+\frac{3 i-5}{2}\right) d z+\left(\frac{-1-5 i}{4}\right) \int_{C} \frac{d z}{z-i / 2}
$$

Applying Cauchy's theorem to $\int_{C}\left(3 z+\frac{3 i-5}{2}\right) d z l(9)$, we have that 
We can also integrate directly $\int_{C}\left(3 z+\frac{3 i-5}{2}\right) d z$ to get zero.

$$
f(a)=f\left(\frac{i}{2}\right)=\frac{1}{2 \pi i} \int_{C} \frac{f(z)}{z-\frac{i}{2}} d z=\left(\frac{-1-5 i}{4}\right)
$$

Next, we divide $3 z+\frac{3 i-5}{2}$ by $z-\frac{i}{2}$ again to $3\left(z-\frac{i}{2}\right)+\frac{6 i-5}{2}$ so that

So that

$$
f(z)=3\left(z-\frac{i}{2}\right)^{2}+\left(3 i-\frac{5}{2}\right)\left(z-\frac{i}{2}\right)+\frac{-1-5 i}{4}
$$

$$
\int_{C} \frac{f(z)}{\left(z-\frac{i}{2}\right)^{2}} d z=\int_{C} 3 d z+\left(\frac{6 i-5}{2}\right) \int_{C} \frac{d z}{\left(z-\frac{i}{2}\right)}+\left(\frac{-1-5 i}{4}\right) \int_{C} \frac{d z}{\left(z-\frac{i}{2}\right)^{2}}
$$

Applying Cauchy's theorem to $\int_{C} 3 d z$ and (9) to $\left(\frac{6 i-5}{2}\right) \int_{C} \frac{d z}{z-\frac{i}{2}} d z+\left(\frac{-1-5 i}{4}\right) \int_{C} \frac{d z}{\left(z-\frac{i}{2}\right)^{2}}$, we have that

$$
\frac{1}{1 !} f^{\prime}\left(\frac{i}{2}\right)=\frac{1}{2 \pi i} \int_{C} \frac{f(z)}{\left(z-\frac{i}{2}\right)^{2}}=\frac{6 i-5}{2}
$$

The given polynomial is $f(z)=3 z^{2}-\frac{5}{2} z+\frac{1}{2}, f^{\prime}(z)=6 z-\frac{5}{2} . f\left(\frac{i}{2}\right)=-\frac{3}{4}-\frac{5 i}{4}+\frac{1}{2}=\frac{-1-5 i}{4}$ and $f^{\prime}\left(\frac{i}{2}\right)=\frac{6 i}{2}-\frac{5}{2}=\frac{6 i-5}{2}$, which is consistent with the values computed above. So far we have obtained the first derivative. To obtain the second derivative, we divide (10) by $z-\frac{i}{2}$ to get

So that

$$
\int_{C} \frac{f(z)}{\left(z-\frac{i}{2}\right)^{3}} d z=\int_{C} \frac{3}{\left(z-\frac{i}{2}\right)} d z+\left(\frac{6 i-5}{2}\right) \int_{C} \frac{d z}{\left(z-\frac{i}{2}\right)^{2}} d z+\left(\frac{-1-5 i}{4}\right) \int_{C} \frac{d z}{\left(z-\frac{i}{2}\right)^{3}}
$$

$$
\frac{1}{2 !} f^{\prime \prime}\left(\frac{i}{2}\right)=\frac{1}{2 \pi i} \int_{C} \frac{f(z)}{\left(z-\frac{i}{2}\right)^{3}}=3
$$

Since our polynomial is of degree two, third and higher derivatives will be zero. This is also easily seen as further division of (12) by $z-\frac{i}{2}$ and integrating will make all terms of the right hand side zero. If we choose a polynomial of degree $n$, then we can divide $n$ times to establish the $\mathrm{n}^{\text {th }}$ derivative.

\section{Example}

As a second example, let us choose $f(z)=z^{4}+z^{3}+z^{2}+z+1$ and follow the same procedure as in the first example. Suppose our $a$ here is 1 . Then dividing $f(z)$ by $z-1$ we get

$$
f(z)=(z-1)\left(z^{3}+2 z^{2}+3 z+4\right)+5
$$

Next we divide $z^{3}+2 z^{2}+3 z+4$ by $z-1$ and get

$$
z^{3}+2 z^{2}+3 z+4=(z-1)\left(z^{2}+3 z+6\right)+10
$$

Further, we divide $z^{2}+3 z+6$ by $z-1$ to arrive at

$$
z^{2}+3 z+6=(z-1)(z+4)+10
$$

Finally, dividing $z+4$ by $z-1$ again, we have

$$
z+4=1(z-1)+5
$$

Writing out $f(\mathrm{z})$ completely by putting (11) to (14) together and dividing by $(\mathrm{z}-1)^{4}$, gives

$$
\frac{f(z)}{(z-1)^{4}}=1+\frac{5}{z-1}+\frac{10}{(z-1)^{2}}+\frac{10}{(z-1)^{3}}+\frac{5}{(z-1)^{4}}
$$

Integrating both sides around $C:|z-1|=r$, we will get at the first division that $f(1)=\frac{1}{2 \pi i} \int_{C} \frac{f(z)}{z-1}=5$. At second division, we get $\frac{1}{1 !} f^{\prime}(1)=\frac{1}{2 \pi i} \int_{C} \frac{f(z)}{(z-1)^{2}}=10$. Also at the third division, $\frac{1}{2 !} f^{\prime \prime}(1)=\frac{1}{2 \pi i} \int_{C} \frac{f(z)}{(z-1)^{3}}=10$. Finally at the fourth division, we find that $\frac{1}{3 !} f^{\prime \prime \prime}(1)=\frac{1}{2 \pi i} \int_{C} \frac{f(z)}{(z-1)^{4}}=5$ To check our results, we compute $f(z), f^{\prime}(z), f^{\prime \prime}(z)$ and $f^{\prime \prime \prime}(z)$. Now $f^{\prime}(z)=4 z^{3}+3 z^{2}+2 z+1$ $f^{\prime \prime}(z)=12 z^{2}+6 z+2, f^{\prime \prime \prime}(z)=24 z+6, f(1)=5, f^{\prime}(1)=10, f^{\prime \prime}(1)=20$ and $f^{\prime \prime \prime}(1)=30$. 
To get the fourth derivative we divide (15) by z -1 again to get that $\frac{1}{4 !} f^{\prime \prime \prime \prime}(1)=\frac{1}{2 \pi i} \int_{c} \frac{f(z)}{(z-1)^{5}}=1$

Higher derivatives are all zero. There is parity between these results.

Generally, we can choose a polynomial of degree $n$ and divide $n$ times following the procedures we have adopted in the examples above to establish the $\mathrm{n}^{\text {th }}$ derivative formula. But before we do that, let's return to examples again to reveal an interesting link between these polynomial division and Taylor's series, from which the Cauchy's integral formula reveal itself.

Now let us pick the second example: Here $f(z)=z^{4}+z^{3}+z^{2}+z+1$

$$
\begin{aligned}
& z^{4}+z^{3}+z^{2}+z+1=(z-1)\left(z^{3}+2 z^{2}+3 z+4\right)+5 \\
& =(z-1)\left[(z-1)\left(z^{2}+3 z+6\right)+10\right]+5 \\
& =(z-1)^{2}\left(z^{2}+3 z+6\right)+10(z-1)+5 \\
& =(z-1)^{2}[(z-1)(z+4)+10]+10(z-1)+5 \\
& =(z-1)^{3}(z+4)+10(z-1)^{2}+10(z-1)+5 \\
& =(z-1)^{3}[1(z-1)+5]+10(z-1)^{2}+10(z-1)+5 \\
& =(z-1)^{4}+5(z-1)^{3}+10(z-1)^{2}+10(z-1)+5
\end{aligned}
$$

Observe that the last equation is the Taylor series expansion of $f(\mathrm{z})$ about $\mathrm{z}=1$ so that the coefficients are exactly the derivatives that appear in Taylor's expansion. What we mean is that

$$
\begin{aligned}
& z^{4}+z^{3}+z^{2}+z+1=(z-1)^{4}+5(z-1)^{3}+10(z-1)^{2}+10(z-1)+5 \\
& =\frac{f^{i v}(1)}{4 !}(z-1)^{4}+\frac{f^{\prime \prime \prime}(1)}{3 !}(z-1)^{3}+\frac{f^{\prime \prime}(1)}{2 !}(z-1)^{2}+\frac{f^{\prime}(1)}{1 !}(z-1)+f(1)
\end{aligned}
$$

So, if we divide both sides by $(z-1)^{5}$ and then integrate both sides around $\mathrm{C}$ such that 1 is in the interior of $\mathrm{C}$, we can easily establish the Cauchy's integral formula for up to the fourth derivative.

\section{Concluding Remarks}

Cauchy's theorem and its integral formula is a very basic result in complex analysis. It is important then to understand the concepts especially that the proof passed through a lot of stages. One of the way is to look at it from various points just like the proofs have different approaches by different people. So in this paper, we have just looked at how we can reach the Cauchy's integral formula from repeated division, which make the theory compare with the elementary ideas of remainder theorem.

The way we arrived at the results looks simpler to understand for beginners than the normal proof of differentiation. As we performed the division repeatedly, we see that we are actually expanding the given analytic function in its Taylor series about the specified point $a \in C$.

This link of Cauchy's integral theorem with Taylor's series is remarkable and who knows whether analysis on Taylor's series as regard convergence and radius of convergence may have link with Cauchy's integral formula. This is a subject for further study.

In a nutshell, we have been able to reach at Cauchy's integral formula, which is not a new result, but from a perspective which is novel.

\section{References}

Ahlfors, L. V. (1979). Complex Analysis.3 edn, USA, McGraw Hill, Inc. 331pp

Alpay, D. (2015). An Advanced Complex Analysis Problem Book 1 edn, Birkhauser Basel, 521pp

Brown, J. W., \&Churchill, R. (2009).Complex Variable and Its Applications.8edn, New York, McGraw Hill, Inc. 468pp

Conway, J. B (1986).Function of One Complex Variable.2edn, New York, Springer Verlag.322pp

Dixon, J. D. (1971). A Brief Proof of Cauchy's Integral Theorem.Proceedings of American Mathematical Society. 29:625-626

Dyer, R. H., \& Edmunds, D. E. (2014).From Real to Complex Analysis.1 edn, Springer International Publishing, Switzerland. $332 \mathrm{pp}$

John, H. M., \&Russell, W. H. (2011).Complex Analysis for Mathematics and Engineering, 6 edn, UK, Jones and Bartlett Learning International. $650 \mathrm{pp}$

Juan, C. P.(2016). Complex Analysis: Problems w i th S o l u t i o n s. https://www.researchgate.net/profile/Juan Ponce_Campuzano/publication/280722238 Complex_Analysis_Problems_with_solut

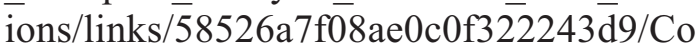
mplex-Analys is - Problems-withsolutions.pdf

McCharty, C. A. (1975). The Cayley - Hamilton Theorem.American Mathematical monthly. 82: $390-391$

Needham, T. (1998).Visual Complex Analysis.1edn, USA, Oxford University Press.592pp

Riemann, B. (1953). The Collected Works of Bernhard Riemann.1edn, New York, Dover Publications, Inc. 704pp

Scott, A. E. (1978). The Cauchy Integral Theorem: A Historical Development of its Proof M.Sc. Thesis, Oklahoma State University.53pp 